\title{
Teorizar la teoría: un debate sobre la independencia epistemológica archivística
}

\author{
Rodrigo Fortes de Ávila* \\ Maria Teresa Navarro de Britto Matos* \\ Miguel Ángel Rendón Rojas ${ }^{\star \star}$
}

Artículo recibido:

3 de diciembre de 2019

Artículo aceptado:

30 de abril de 2020

Artículo de investigación

\section{Resumen}

Se analizan las discusiones que tuvieron lugar en las revistas American Archivist y Archivaria entre 1982 y 1999 que reflejan las luchas de la archivística por su identidad como ciencia autónoma. Es posible identificar tres movimientos epistemológicos. El primero apunta la teoría como obstáculo, critica la ineficacia de las generalizaciones científicas, su búsqueda revela más una necesidad del reconocimiento social de la profesión. El segundo se refiere a la teoría como fundamento de cientificidad, que reconoce propiedades esenciales ligadas a la naturaleza de su objeto de estudio y la necesidad de estudiarlas y protegerlas. El último aborda la

\footnotetext{
* Instituto da Ciências da Informação. Universidade Federal da Bahia, Brasil rodrigo.avila@ufba.br_maria.navarro@ufba.br ** Instituto de Investigaciones Bibliotecológicas y de la Información, UNAM. México

marr@unam.mx INVESTIGACIÓN BIBLIOTECOLÓGICA, vol.35, núm. 86, enero/marzo, 2021, México, ISSN: 2448-8321 pp. 13-28
} 
teoría como transgresión, es decir, como una herramienta de transformación que debe producir cambios indispensables en el desarrollo del campo y considerarla como una forma de liberar las restricciones impuestas por la tradición.

Palabras clave: Archivos; Archivística; Epistemología; Teoría

Theorizing the theory: a debate on archival epistemological independence

Rodrigo Fortes de Ávila, Maria Teresa Navarro de Britto Matos and Miguel Ángel Rendón Rojas

\section{Abstract}

The discussions that took place in the American Archivist and Archivaria between 1982 and 1999, are hereby analyzed and reflect the struggles of the Archival Science for finding its identity as an autonomous science. It is possible to establish here three epistemological shifts. The first one points to the theory as an obstacle, criticizing the uselessness of scientific generalizations, and its search reveals more a need for social recognition of the profession. The second one refers to the theory as a foundation of scientificity which recognizes essential properties as linked to the nature of its object of study and the need to study and protect these. The last of them approaches the theory as transgression, that is, as a transformation tool that must produce indispensable changes in the development of the field and consider this a way to release the restrictions imposed by tradition.

Keywords: Archives; Archives Studies; Epistemology; Theory

\section{INTRODUCCIÓN}

Doco después de la publicación del Manual Holandés (Muller, Feith y Fruin, 1898), cuyas propuestas estaban orientadas por el principio de procedencia, surgieron discrepancias sobre el status de la teoría archivística. Cruz Domínguez (2017) afirma que desde ese momento surge la defensa de 
la autonomía del campo como una respuesta de los archivistas a las críticas de los historiadores holandeses sobre los principios establecidos en dicho manual.

Ese debate sobre la "soberanía" archivística ha ayudado a la consolidación de la identidad del campo. La lucha por la emancipación es tan evidente que, noventa años después de la publicación del Manual, Lodolini escribe “The War of Independence of Archivists" (1989). En 1929, la recesión económica en los Estados Unidos, así como los trabajos de la comisión Hoover a finales de la década de los 40, fungieron como fundamentos de la teoría de records managements, la cual, aunada a los principios de evaluación de Schellenberg (1956), terminan por incrementar la tensión con la Historia. Los historiadores criticaban el hecho de que el principio de procedencia era insuficiente porque respondía estrictamente a los valores gubernamentales.

Metodología. El presente trabajo se circunscribe dentro de esa temática epistemológica sobre la identidad de la Archivística, y está guiado por las preguntas ¿cuáles han sido las principales posturas epistemológicas que han respondido a esa problemática?, ¿es posible hablar sobre una direccionalidad en el devenir de la disciplina, guiado por una propuesta metateórica que permita abrir el camino a su desarrollo en contraposición a un estancamiento, si se siguen otros derroteros? Por consiguiente, los objetivos de este artículo en concordancia con las interrogantes planteadas son analizar las principales posturas epistemológicas propuestas como respuestas a la identidad de la Archivística, y valorar una propuesta metateórica que permita abrir el camino a su desarrollo. Las hipótesis que se desprenden de los problemas investigados son que existen tres modelos que fundamentan la identidad disciplinaria de la Archivística: una propuesta tecnicista pragmática acientífica; un enfoque cientista, eminentemente fundamentado en el positivismo según el cual la ciencias naturales se convierten en el arquetipo de toda ciencia, es decir, la ciencia es empírica, cuantificable, nomológica, instrumentalista, pseudobjetiva, sin compromiso social, y un enfoque que se fundamenta en una concepción más amplia que la positivista y da cabida a las ciencias sociales y humanas con una visión crítica sociocultural. Este último enfoque abre el horizonte para el desarrollo de la Archivística, permite trabajar la documentación de las minorías, lo que posibilita escribir la "historia de los hombres infames"; de lo contrario, si se permanece en lo técnico, y en el modelo positivista donde nació la disciplina, se continuará con la gestión de los documentos administrativos, aprobados por la visión de Estado dominante, contribuyendo a redactar "la historia de los hombres ilustres". La metodología empleada es la acorde con este tipo de estudios abstractos, incluye el método hermenéutico para interpretar las propuestas 
de los autores consultados, el análisis y el método deductivo para justificar nuestros razonamientos.

El material empírico sobre el que versará nuestro análisis y que sirve de delimitación espacio-temporal del problema son los debates que se realizaron entre 1982 y 1999 en las revistas American Archivist y Archivaria en torno a la necesidad de una teoría archivística que guie las acciones profesionales. La razón por la que se eligieron los artículos de esas revistas es que reflejan de manera condensada las posturas de los archivistas sobre la problemática y nos sirven para fundamentar nuestras hipótesis, de tal manera que nuestro trabajo no es meramente descriptivo-narrativo historiográfico, sino teórico-analítico.

Este artículo se divide en tres partes que reflejan tres movimientos epistemológicos presentes en esa disputa. El primero explora la teoría como un obstáculo. Se cuestiona la necesidad de una base teórica universal para el campo que vaya más allá de los principios prácticos. Asimismo, se critica la ineficacia de las generalizaciones científicas; su búsqueda revela más bien un esfuerzo de autonomía por la necesidad psicológica de reconocimiento social de la profesión.

La segunda parte investiga la teoría como fundamento de cientificidad, hasta cierto punto, como redención. En ella, se defiende la relevancia de la abstracción para proteger las propiedades esenciales del objeto de estudio. La cuestión central es entender la función de la teoría y cómo el campo desarrolla su base epistemológica.

El último apartado aborda la teoría como transgresión. A partir de la década de 1990 surge un movimiento que concibe la teoría como una herramienta de transformación que evalúa "por qué hacemos lo que estamos haciendo".

\section{LA TEORÍA COMO OBSTÁCULO}

Las anteriores posiciones son respuesta a las provocativas reflexiones de Frank Burke. La idea general de su argumento es que la teoría debe verse como "desarrollo de leyes universales e inmutables que deben aplicarse en todo momento, independientemente del tiempo y el lugar" (Burke, 1981: 40). Desde esa perspectiva, los principios archivísticos de respeto a la procedencia y respect des fonds serían ejemplos típicos de leyes prácticas que tuvieron éxito en su aplicabilidad.

Ese esfuerzo de "teorizar la teoría" condujo a Burke a declarar que la mayoría de los escritos archivísticos son principios prácticos para profesionales. Concluye que, si la "teoría" no es útil para ayudar a la resolución de los 
problemas cotidianos, entonces su necesidad debe ser cuestionada. De esta manera, clama por encontrar algo más amplio, no sólo las interminables discusiones sobre los documentos archivísticos, sino un debate sobre su naturaleza como registros humanos.

El historiador Lester Cappon (1982) evalúa más detalladamente la división "burkeniana" entre los principios prácticos profesionales y las leyes teóricas universales. Para él los principios orientadores despreciados por Burke deben considerarse la base teórica de la profesión debido a sus resultados, modelando reglas específicas que han sido resignificadas a lo largo del tiempo.

Toda esta reflexión sobre la esencia de los registros humanos lleva a Burke a argumentar que los estudios universitarios de archivo son entrenamientos bajo el disfraz de la educación archivística donde "los alumnos aprenden qué y cómo, pero no por qué” (Burke, 1981: 44-45). Por esta razón, su voz expresa la necesidad de leyes universales en la teoría archivística para comprender la práctica de los profesionales.

$\mathrm{Al}$ año siguiente, las inquietudes "burkenianas" llevan a Cappon (1982) a publicar un artículo extremadamente relevante: "What, then, is there to theorize about?". El texto en sí ya es una pregunta retórica sobre la propia esencia de la cuestión. ¿Cuáles son las implicaciones de que la teoría archivística no sea sobre la propia práctica en los archivos? Desde este punto de vista, la teorización propuesta por Burke compromete la función principal del archivista de ser, en las propias palabras de Cappon (1982: 19), un "guardián de los registros". Además, no sería una buena idea ingresar al "reino de los historiadores", reiterando el pensamiento de Jenkinson de que "el archivista no es y no debería ser un historiador" (citado por Cappon, 1982: 22) y protege la "santidad" de la evidencia como "devoto más desinteresado de la verdad”. En la opinión de Cappon (1982), en el aula los estudiosos aprenden a formular esas leyes universales y la teoría surge "en las condiciones contemplativas del claustro" académico (Burke, 1981: 42). Esta misma visión asumirá Theo Thomassen (1999), para quien la defensa de la autonomía a través de la libertad académica elimina subordinaciones institucionales que debilitan a la disciplina.

Con respecto al dilema entre la Historia y los archivos presentado por Burke, Cappon (1982) afirma la existencia de una "verdad archivística" diferente de la histórica. Mientras que en la última la preocupación está en el contenido de los textos, en la primera lo es la autenticidad y la preservación continua de los registros. Con esos argumentos, Cappon presenta un punto de vista más cauteloso al sugerir que debe observarse una conducta más conservadora de lo que proyecta Burke, ya que sumergirse en una abstracción profunda conlleva el peligro de producir cambios bruscos, comprometiendo tal "verdad archivística". 
Para combatir los argumentos sobre la necesidad de una teoría archivística que descubriría leyes universales y explicaría "los porqués", Roberts escribe una serie de artículos en los cuales examina los razonamientos a favor de la teorización, concluyendo que la teoría archivística definida por Burke resulta innecesaria. Comparando las funciones de los archivistas con las de los periodistas, afirma que su éxito no radica en los contenidos que tratan, sino en las técnicas que se aprenden por la práctica diaria. Este paralelo retrata el trabajo en los archivos como algo sencillo que no justifica la necesidad de una teoría que "complica lo que es simple". Por esta razón, los porqués "burkenianos" pondrían a los teóricos del campo en una situación contraproducente, ya que parece "improbable que tales cuestiones rendirían mucho para avanzar el trabajo en los archivos" (Roberts, 1990: 110, 117).

La discusión de Roberts se remonta a un artículo de 1987, donde parece repetir las ideas de Cappon al denunciar el afán de una teoría archivística universal. En ese trabajo señala que la teoría de los archivistas se reduce a guardar materiales de interés histórico con el propósito de proteger la naturaleza de los archivos, cuyo valor último reside en ser fuentes de investigación (Roberts, 1987).

Ese discurso, fundamentalmente "esencialista" señala que la teoría de los archivos jamás podrá estructurarse con validez universal, porque esos acervos no tienen características comunes. Al contrario, representan una variedad infinita de materiales y singularidades. En consecuencia, no hay necesidad de conocimiento teórico porque dicho trabajo puede traducirse empíricamente. Por lo tanto, "no hay una imagen grande en los archivos, solo un número infinito de imágenes pequeñas que se pueden capturar por separado" (Roberts, 1990: 112). Del mismo modo en que Simone de Beauvoir destacó la ausencia de una universalidad humana, cuya esencia está en no tener esencia, Roberts afirmó que en el campo archivístico no hay esencias, la teoría debería preocuparse únicamente por los problemas relacionados con las técnicas y los procedimientos. Para el investigador, estos insights de orientación práctica son más provechosos que las estériles nociones teóricas. De esta manera, es mejor reflexionar sobre lo específico. Al intentar abarcar aspectos que van más allá de los procedimientos mecánicos, buscando sistemas integrales, su utilidad se arruinaría. Según la opinión de Roberts, tales intentos desean imponer precisión en un campo de extrema inexactitud científica.

Semejantes razonamientos fortalecen el desinterés de Roberts por leyes universales. Con su preocupación restringida a normas que orientan actividades diarias, justifica la inutilidad de la teoría con el hecho de que "los archivos contienen informaciones históricamente valiosas o no" (Roberts, 1990: 112). Su conclusión nos coloca en una posición vulnerable porque la 
única teoría del campo sería salvar "lo que es históricamente valioso" (Roberts, 1987: 70).

Años después, Roberts (1994a) profundiza sus reflexiones en un texto devastador. Su examen se hace más agudo mediante una crítica a la necesidad teórica que sólo imita métodos académicos sin la preocupación en producir estudios valiosos. Según él, la teoría produce la ilusión del profesionalismo al ofrecer generalizaciones científicas que finalmente resultan ser contraproducentes.

Esta misma justificación se plantea con respecto a la historicidad de la construcción del pensamiento archivístico. Roberts (1994a) afirma que sería muy provechoso que los archivistas lo aprendan. Sin embargo, la comunidad estaría equivocada al creer que reflexionar sobre la historia de la administración pública es relevante como orientación profesional. Continúan realizándose trabajos teóricos, generalmente basados en conocimientos prácticos de registros específicos, sobre el oficio práctico de solucionar problemas cotidianos. Sin embargo, se ofrecen sólo como directrices opcionales para evitar la presunción de que se construyen esquemas generales.

En ese mismo artículo Roberts describe una justificación psicológica para el anhelo teórico del campo. La ambición de teorizar refleja la desesperación causada por su bajo reconocimiento social. Para una profesión acostumbrada a ocupar el "bajo clero" de la jerarquía organizacional burocrática, la teoría se revela como una tabla salvadora que pretende ocultar la debilidad de su imagen social. Es por ello que los archivistas debaten cuestiones teóricas, no por avanzar en la comprensión de su objeto de estudio, sino para tener una herramienta que subsidia la ilusión del autoreconocimiento. Sólo el "salto teórico" llevaría a una "situación de igualdad" con los historiadores, porque la sistematización teórica los incorporaría a un campo de estudio más prestigioso. Los cuestionamientos acerca de la naturaleza de los registros y de la "verdad" de los hechos pertenecen únicamente al terreno de la Historia. La búsqueda por teorización es más bien una "necesidad emocional de una mayor aceptación profesional” (Roberts, 1987: 67) y su interés indicaría un desdoblamiento del complejo de inferioridad. En resumen, la teoría sería una herramienta subordinada a objetivos egoístas por "campañas publicitarias" disfrazadas de exploraciones académicas. Pero no se detiene ahí. Señala que la teoría desempeña un papel primordial en la lucha por la definición de las agendas, impulsando la expansión profesional. En este sentido, los teóricos buscan analizar cuestiones que no requieren profundización, desarrollan modelos que nada elucidan y esclarecen conceptos autoevidentes. Para el investigador estadounidense, la subordinación profesional a los teóricos es velada; los científicos ganadores son los verdaderos orientadores de la dirección de los debates. 
Las críticas de Roberts declaran que la teorización en los archivos es buscar el glamour. La búsqueda por estándares revela el esfuerzo por establecer la independencia y estatus social, desvelando una lucha política que determina qué grupos orientan las trayectorias profesionales y promoviendo el control de las universidades en la formación profesional. Para Roberts, el equívoco de esta postura no es tanto desconsiderar que los conceptos archivísticos "no son lo suficientemente válidos como expresiones culturales para ser estudiadas académicamente" (Roberts, 1990: 113), sino no señalar que elucidan un mecanismo cuya aplicación es limitada. Además, los conceptos archivísticos existen para facilitar el estudio de otras disciplinas. Por lo tanto, el campo no tiene vigor intelectual verdaderamente independiente. Eso muestra que hasta el momento las cosas no se están haciendo correctamente.

\section{LA TEORÍA COMO FUNDAMENTO DE CIENTIFICIDAD}

En el mismo año que Roberts escribe tales ideas, Terry Eastwood publica "What is archival theory and why its important?" en la revista canadiense Archivaria. El autor manifiesta que la noción de "verdad" presupone la contemplación de un objeto para comprender su naturaleza a través de la explicación de sus propiedades. Esa acción implica ideas sistematizadas que aclaran fenómenos, expresadas en una proposición. De esta manera, la teoría no es una suposición que origina la contemplación; al contrario, es más bien una construcción mental derivada de la observación para elucidar la naturaleza del objeto. Si el método y la práctica se basan en la teoría, entonces ellos pueden convertirse en laboratorios para el desarrollo de la misma teoría.

Así pues, Eastwood (1994) afirma que la teoría consiste en la elaboración de ideas sobre los objetos contemplados y el método indica cómo proceder para observarlos, mientras que la práctica apoya la aplicación teórica con el fin de ampliar el conocimiento sobre el producto analizado. En controversia con Roberts, el canadiense afirma que toda disciplina aplicada se basa en algún cuerpo de conocimiento abstracto. La cuestión central del debate no es si la teoría en estos campos es algo contraproducente, sino qué parte toma en esa construcción y cómo desarrolla su base de conocimiento. Desde este punto de vista, el propósito de la teoría archivística consistiría en elucidar la naturaleza del material en sí, que dejaría de ser un objeto exclusivo del campo histórico. En una visión que actualmente sigue Luciana Duranti (1996), quien reitera que el primer objeto de la teoría es la evaluación de la naturaleza de los registros y la protección de sus propiedades de evidencia. La gran pregunta teórica se resumiría como ¿qué propiedades deben protegerse y por qué razones? 
Eastwood (1994) señala que "rendir cuentas" es un valor intrínseco de la naturaleza de los archivos, lo que los convierte en artefactos que posibilitan el conocimiento de las experiencias humanas. Debido a que los documentos son herramientas esenciales para conducir los negocios, las raíces de la teoría se remontan a principios legales y administrativos. En estos términos, cada archivo permanece como evidencia de transacciones comerciales, proporcionando medios para una mayor investigación. El investigador canadiense certifica que la teoría se sostiene en dos bases: 1) los documentos dan fe de hechos y actos; 2) su fiabilidad depende de las circunstancias de los contextos de producción y preservación a lo largo del tiempo.

Desde su perspectiva epistemológica, Eastwood (1994) defiende al campo de los ataques de Roberts por su excesiva preocupación por el método y la práctica. ¿Acaso esta profesión sólo estaría siendo subsidiada por el método y la práctica, sin ninguna reflexión sobre sus intervenciones? Desde su punto de vista, los pensadores del campo se respaldaron en la experiencia como un esfuerzo consciente de preservación de las fuentes del pasado en instituciones dedicadas a este propósito. En la perspectiva moderna, los archivos eran caracterizados como conjuntos producidos por organizaciones o personas en el curso de sus actividades. Eso hizo que sus reflexiones se vincularan a los medios de tratamiento de los documentos y no a las cuestiones referentes a sus características. Ese equívoco provocó que se olvidara que comprender esos atributos genera características universales, las cuales sustentan la calidad de los archivos, haciendo que el método y la práctica funcionen para proteger su esencia dirigidos por un juicio teórico.

Desde esta perspectiva, las cualidades que deben protegerse son imparcialidad, ${ }^{1}$ autenticidad, ${ }^{2}$ naturalidad, interrelación y unicidad. ${ }^{3}$ Dichas características guían los caminos para mantener el significado esencial de los archivos como valor de evidencia. Para Eastwood, comprender las relaciones funcionales y estructurales que encapsulan su génesis protege la integridad de tales conjuntos.

1 Elaborados como instrumentos que expresan acciones cotidianas, dichos registros se convierten en productos inmunes a intereses posteriores. Sus circunstancias de génesis aseguran que no fueron escritos visualizando la posteridad.

2 Predice que deben estar protegidos por procedimientos regulares que puedan ser atestiguados legal y administrativamente. Indica que el propósito de la teoría va más allá de los propios registros, cubriendo los contextos procesales tanto de su generación como de su preservación.

3 La relación intrínseca entre documento y demanda reitera el hecho de que son interdependientes tanto en su significado como en su capacidad para servir como evidencia. La teoría va a concentrarse en este vínculo directo entre la "actividad funcional-documentos", insertada en una estructura administrativa, donde cada documento tiene un lugar único en la formación de un archivo. 
Este pensamiento reivindica para la Archivística un estatus de autonomía con dimensiones teóricas, metodológicas y prácticas que apoyan un cuerpo coherente de conocimiento. El punto de partida es la teoría que generaliza la naturaleza de los archivos, determina las características comunes y define un fundamento intelectual para el método y la práctica. Estas particularidades constituyen la teoría orgánica de los archivos, definiendo el quehacer y desarrollo del campo. Como "protectores de la evidencia", dichos profesionales aseguran que las relaciones sociales se pueden explicar con base en esas evidencias. Obviamente, el argumento sobre qué son los hechos reales no se puede evitar en el análisis orgánico. Sin embargo, todos los juicios proceden de la calidad de las evidencias; se evalúa a través de luces teóricas que reflejan las circunstancias condicionantes de su génesis. Por estas razones, Eastwood sostiene que las controvertidas reflexiones de Roberts perjudican el concepto de "teoría archivística" al olvidar el propósito racional de concebir métodos que protegen sus características elementales, con la intención de promover el futuro uso confiable de las fuentes de investigación.

En la década de 1990 la discusión sobre este tópico sube de nivel, y en la revista Archivaria Roberts (1994b) responde las notas de Eastwood (1994). El pensador estadounidense se defiende de un malentendido, según él, presentado en los argumentos del canadiense: se le acusa de no reconocer la existencia de una teoría archivística. Sin embargo, esa apreciación no es correcta. Reformulando su razonamiento, Roberts declara que no se trata de declarar la imposibilidad de construir abstracciones universales. El punto neurálgico de su argumento es la crítica a los fundamentos por los cuales Eastwood determina que la teoría archivística no debe separarse de la objetividad de las interpretaciones del contenido, donde los trabajos teóricos criticados son aquellos que "intentan facilitar la objetividad interpretativa o que intentan envolver el contenido con fórmulas generales" (Roberts, 1994b: 131). Es decir, considera erróneo proporcionar respuestas metodológicas a los dilemas relacionados con el contenido, de tratar de hacer lo imposible.

Además, Roberts (1994b) considera que los profesionales en el campo no pudieron proteger la documentación sobre cuestiones sociales relevantes, no por discapacidad intelectual, sino por las herramientas conceptuales disponibles para evaluar los registros, basadas en las propiedades presentadas por Eastwood, ineficaces para seleccionar materiales representativos.

Finalmente, Roberts (1994b: 133) concluye con una posición más conciliadora:

El profesor Eastwood y yo estamos de acuerdo en la mayoría de las cuestiones. Es- 
alguna teoría archivística tiene fallas; estamos de acuerdo en que alguna teoría archivística es válida; y estamos de acuerdo en lo que constituye una teoría archivística defectuosa y válida. Con la excepción del desacuerdo sobre la importancia de la teoría válida para la misión archivística general, nuestras diferencias en relación con la teoría archivística parecen ser tan insignificantes como la propia teoría archivística.

\section{LA TEORÍA COMO TRANSGRESIÓN}

Además de esa visión teórica de la Archivística que busca aclarar la naturaleza de su objeto de estudio, a partir de la década de 1990 aparece otra que bosqueja la teoría como una herramienta de transformación. En ese sentido, Frederick J. Stielow libera al archivista de ese "juicio tradicional”, dejándolo libre para "vuelos teorizantes" al presentar la relevancia de la teoría para comprender sus profesionales como intermediarios del "proceso de información archivística y en el continuum entre fuentes y usuarios” (Stielow, 1991: 26).

Ese enfoque menos restrictivo se alinea a la visión de las Ciencias Sociales según la cual las teorías deben utilizarse para intervenir en la realidad, convirtiéndose en instrumentos para provocar cambios en el desarrollo del campo. Stielow parte de la noción de que la realidad de los archivos no es ajena a la transformación temporal. Por lo tanto, la teoría sirve para señalar que siempre habrá espacio para el desarrollo de nuevos principios, porque su formulación refuerza la idea que "los seres humanos, que crearon la institución y sus prácticas, tienen el poder de modificarlas” (Stielow, 1991: 23). Nunca se ha podido distinguir la dirección que tomamos sin cuestionar los caminos que deben seguirse. En resumen, lo que puede comprenderse por la teoría es cómo evaluar, por qué hacemos lo que estamos haciendo.

Dentro de esta perspectiva, Nicholas Graham (1997) sostiene que teorizar no se puede observar como un privilegio o como un lujo. La teoría en este campo es, antes que nada, sustancial, porque las informaciones contenidas en los archivos son partes constitutivas de la sociedad. Su existencia jamás podrá considerarse como un vacío. Esta permeabilidad preconiza una exploración política, histórica, social y cultural para que se mantengan relevantes como instituciones sociales.

Mientras Burke (1981) y Roberts (1987; 1990; 1994a) consolidaron una perspectiva estrictamente histórica en los archivos, Graham (1997) pondera el porqué de esta noción de validez existencial. Para él, la interpretación de Roberts se basa en lo que los profesionales hacen o dejan de hacer en lugar de cuestionar si la teoría es necesaria o incluso útil para el campo. La teoría 
debe involucrar esfuerzos para comprender en qué medida debe emplearse, llevándonos a métodos y estructuras, y no exaltar la práctica por la práctica, disminuyendo el valor de la experiencia del conocimiento como base indispensable del hacer. Esto se debe a que las reglas indican cómo guiar las acciones, mientras que la teoría pregunta los porqués, liberándose de las limitaciones de sus posibilidades reales. Por lo tanto, la teoría no puede presentar respuestas simplistas, mucho menos explorar ideas sin la obsesión por una justificación. En resumen, ellas no deben ser observadas mientras amenazan a los archivos. Son necesarias para abrir espacio a una discusión sobre la propia claridad de la existencia de las cosas.

Roy C. Schaeffer destaca la relevancia de los archivos para la "sociedad en su conjunto". En su evaluación, el archivista asume el papel de "servidor social" con el deber de "proteger los derechos de la comunidad mundial y la capacidad de entender y evaluar el funcionamiento de las instituciones y su propia evolución en un contexto histórico integral" (Schaeffer, 1992: 615). Sería necesario examinarlos a la luz de sus implicaciones sociales para comprender la función de la teoría en la formulación de nuevos principios.

La aceptación de este papel de intermediario en las demandas sociales implica asumir la responsabilidad de definir una base conceptual para las acciones. En términos generales, Schaeffer (1992) nos recuerda que las reflexiones del campo siempre exploran los registros: ¿qué son?, ¿cuáles deben conservarse y cómo deben organizarse? Esto hace que uno considere a las instituciones sólo como los organismos que las crearon, no como organizaciones sociales. De hecho, lo que propone el investigador es dar un paso atrás para expresar nuevos conocimientos sobre su función social.

Anclado en el campo minado de la memoria colectiva, Foote (1990) examina los archivos como residuos de la misión institucional de las organizaciones sociales. En su pensamiento, para mantener un papel destacado en la formación de la memoria colectiva, sería extremadamente necesario determinar dónde están las informaciones consideradas relevantes para las generaciones futuras. Este es, exactamente, y si quieren funcionar con primacía en la preservación de la memoria social del área, el juego discursivo que los profesionales necesitan esforzarse por comprender. Esta comprensión resalta el entendimiento de los conflictos y los intentos de cualquier comunidad de lidiar con las tensiones de su pasado y proyectarse a su futuro.

\section{Conclusiones}

Se puede descubrir en las lecturas de los textos presentados que las inquie- 
estimularon la investigación teórica en la década de 1980, aunque, como ya se indicó al principio y citando a Cruz Domínguez (2017), esa inquietud y la lucha por su autonomía teórica se remontan a mucho tiempo atrás, casi desde su misma aparición. Debido a que en sus reflexiones trabajó con el argumento de que no hay "esencia archivística" para resolver dilemas prácticos particulares, las generalizaciones científicas parecían contraproducentes. Esa visión retrató el primer camino del texto, en el cual su posicionamiento teórico levantaría una barrera al tratar de imponer precisión en un campo de extrema inexactitud científica. Asimismo, alegó equívocos en su intención de proporcionar respuestas metodológicas a los dilemas vinculados a la gestión del contenido de los documentos. Su búsqueda sería una especie de glamourización de algo simple.

Según sus críticos, este pensamiento distorsionó la comprensión de la naturaleza de los documentos al menospreciar la intencionalidad racional de concebir métodos que protegieran sus características elementales. Eastwood recurrió al argumento de que la teoría es un elemento salvador de la cientificidad al predecir ideas sistematizadas que aclaran fenómenos a través de una(s) propocición(es). La teoría como construcción mental derivada de la observación clarifica la naturaleza del objeto. Por esta razón, el canadiense se preocupó por la protección de propiedades específicas a fin de promover el uso fiable de las fuentes.

Por otro lado, en el contexto de los artículos analizados, a partir de la década de 1990 es posible ver la concepción de la teoría que hemos denominado como transgresión. Los aspectos culturales relacionados con la constitución de los marcos teóricos comienzan a ganar relevancia. En esta perspectiva, las percepciones de Burke no pertenecerían a la teoría propiamente dicha, sino a cuestiones culturales derivadas de la evaluación de los documentos. Como señala Kimball (1985), la teoría comienza a tomar el sentido de un acto de interpretación de su propio recorrido histórico.

Desde nuestro punto de vista, la riqueza teorética de los debates analizados reside en que en su unidad manifiestan claramente las posiciones epistemológicas que se pueden adoptar sobre la autonomía de la Archivística como disciplina científica. Por supuesto que otros autores, en otras latitudes y en diferentes momentos, incluso tal vez anteriores a las fechas de los textos aquí analizados, discutieron las mismas cuestiones; pero el hilo rector que seguimos con los autores citados nos lleva a cierta regularidad a las respuestas propuestas. En un primer término se presenta la visión tecnicista, instrumentalista y pragmática que considera la teoría como innecesaria o incluso perniciosa. En un segundo momento, como reacción a la anterior, se reclama la necesidad de una teoría que fundamente la práctica. Sin embargo, las 
argumentaciones en pro o en contra la teoría giran en torno a una concepción positivista de la ciencia, ya que se le considera empírica (la teoría se deriva de la observación y experimentación), y descubre leyes universales e inmutables. No se toman en cuenta las discusiones sobre la dependencia de la observación con respecto a la teoría: los enunciados observacionales presuponen la teoría, la teoría guía la observación y la experimentación (Chalmers, 2001: 39-58) y la existencia de ciencias, como las humanas o sociales, que no necesariamente descubren leyes. Finalmente, se propone una visión social y crítica que supera la posición positivista de la ciencia, lo que muestra la confirmación de nuestras hipótesis enunciadas al principio del texto.

Es interesante constatar que a una conclusión semejante sobre la evolución de la ciencia de la información (CI) llegaron Rafael Capurro y Birger Hjørland, para quienes la historia de esa ciencia también desemboca en una visión social. Capurro (2007) habla de los ya conocidos tres paradigmas de la CI: físico, cognitivo y social, mientras que para Hjørland (2017) son seis las etapas por las que ha pasado dicha ciencia: paradigma ateórico, la teoría de la información, la tradición de Cranfield, la visión cognitiva, la filosofía de la información de Floridi, y las visiones socioculturales.

El comprender la Archivística como instrumento transformador dejó ver la función social de los archivos, uniéndolos al desarrollo del pensamiento crítico. Tal visión reiteró que los beneficios de teorizar, pero no desde una posición positivista, son indispensables para el avance del campo al abrir posibilidades de transformación. Es cada vez más presente el pensamiento de que el perfeccionamiento de una base teórica coherente prepara el camino para que los archivos sean considerados elementos sociales indispensables.

Se puede observar que, a pesar de su preludio de autonomía débil, la teoría disciplinaria aún tiene un largo camino por recorrer. En vista de esto, las cuestiones planteadas en los debates analizados siguen siendo actuales, incomodando el campo de investigación. Por supuesto, las molestias ocasionadas por los cuestionamientos preservan el diálogo intelectual. De este modo, la inquietud siempre servirá como una herramienta indispensable para mantener la plenitud de la función social de los archivos.

\section{REFERENCIAS}

Burke, Frank G. 1981. "The future course of archival theory in the United States". American Archivist 44 (1): 40-46. https://doi.org/10.17723/aarc.44.1.4853801307551286 
Cappon, Lester J. 1982. "What, then, is there to theorize about?". American Archivist 45 (1): 19-25. https://doi.org/10.17723/aarc.45.1.q03v972668401056

Capurro, Rafael. 2007. “Epistemologia y ciencia de la información”. Enl@ce: Revista Venezolana de Información, Tecnología y Conocimiento 4 (1): 11-29. Fecha de consulta: 17 de marzo de 2020 . https://produccioncientificaluz.org/index.php/enlace/article/view/13372/13357

Chalmers, Alan. 2001. ¿Qué es esa cosa llamada ciencia?. México: Siglo XXI.

Cruz Domínguez, Silvana Elisa. 2017. "La permanente defensa de la archivística por su autonomía”, en La Archivística y la Ciencia de la Información documental: autonomía e interdependencias, coordinado por Miguel Ángel Rendón Rojas, 39-58. México: UNAM. Instituto de Investigaciones Bibliotecológicas y de la Información.

Duranti, Luciana. 1996. Diplomática: usos nuevos para una antigua ciencia. Sevilla: S\&C ediciones.

Eastwood, Terry. 1994. "What is archival theory and why is it important?". Archivaria (37): 122-130. Fecha de consulta: 10 de enero de 2020.

https://archivaria.ca/index.php/archivaria/article/view/11991/12954.

Foote, Kenneth E. 1990. "To remember and forget: Archives, memory, and culture". American Archivist 53 (3): 378-392. https://doi.org/10.17723/aarc.53.3.d87u013444j3g6r2

Graham, Nicholas M. 1997. "The Form and The Function of Archival Theory”. The Katharine Sharp Review (4): 1-7. Fecha de consulta: 10 de enero de 2020. https://www.ideals.illinois.edu/bitstream/handle/2142/78249/graham_form. pdf? sequence $=2$ \& is Allowed $=y$

Hjørland, Birger. 2017. "Library and information science (LIS)". ISKO Encyclopedia of Knowledge Organization. Fecha de consulta: 17 de marzo de 2020. https://www.isko.org/cyclo/lis.htm

Jenkinson, Hilary. 1922. A manual of archive administration. Oxford: Clarendon Press.

Kimball, Gregg D. 1985. "The Burke-Cappon debate: Some Further criticisms and considerations for archival theory". American Archivist 48 (4): 369-376. https://doi.org/10.17723/aarc.48.4.lt0k78j250488v16

Lodolini, Elio. 1989. "The War of Independence of Archivists”. Archivaria (28): 3647. Fecha de consulta: 10 de enero de 2020. https://archivaria.ca/index.php/archivaria/article/view/11569/12515

Muller, Samuel, Johan Feith y Robert Fruin. 1898. Handleiding voor het ordenen en beschrijven van archieven: ontworpen in opdracht van de Vereeniging van Archivarissen in Nederand. Groningen: Erven B. van der Kamp.

Roberts, John W. 1987. "Archival theory: much ado About shelving". American Archivist 50 (1): 66-74. https://doi.org/10.17723/aarc.50.1.1357257455776g52

Roberts, John W. 1990. "Archival theory: Myth or banality?". American Archivist 53 (1): $110-120$. https://doi.org/10.17723/aarc.53.1.a56364w710276424

Roberts, John W. 1994a. "Practice makes perfect, theory makes theorists". Archivaria (37): 111-121. Fecha de consulta: 10 de enero de 2020. https://archivaria.ca/index.php/archivaria/article/view/11990/12953.

Roberts, John W. 1994b. "Response to Terry Eastwood Paper". Archivaria (37): 131133. Fecha de consulta: 10 de enero de 2020. https://archivaria.ca/index.php/archivaria/article/view/11992/12955 
Schaeffer, Roy C. 1992. "Transcendent concepts: Power, appraisal, and the archivist as 'social outcast". American Archivist 55 (4): 608-619. https://doi.org/10.17723/aarc.55.4.g8x4677115671880

Schellenberg, Theodore R. 1956. Modern Archives. Principles and Techniques. Chicago: University of Chicago Press.

Stielow, Frederick J. 1991. "Archival theory redux and redeemed: Definition and context toward a general theory". American Archivist 54 (1): 15-26. https://doi.org/10.17723/aarc.54.1.k84780387v18358k

Thomassen, Theo. 1999. "Archivists Between Knowledge and Power on the Independence and Autonomy of Archival Science and the Archival Profession". Arbivski vjesnik (42): 149-167. Fecha de consulta: 10 de enero de 2020.

https://hrcak.srce.hr/10590

Para citar este texto:

Fortes de Ávila, Rodrigo, Maria Teresa Navarro de Britto Matos y Miguel Ángel Rendón Rojas. 2021. "Teorizar la teoría: un debate sobre la independencia epistemológica archivística”. Investigación Bibliotecológica: archivonomía, bibliotecología e información 35 (86): 13-28.

http://dx.doi.org/10.22201/iibi.24488321xe.2021.86.58228 